\title{
Technological supporting performance of machines and mechanisms
}

\author{
Oleg Burlachenko ${ }^{1}$, Yuri Serdobintsev ${ }^{1}$, and Alexander Lyashenko ${ }^{1, *}$ \\ ${ }^{1}$ Volgograd State Technical University, 400005 Volgograd, Russian Federation
}

\begin{abstract}
The problem of the machines and mechanisms working capacity technological support is considered in this article. The existing methodologies for ensuring operability were analyzed here. The authors revealed and justified the existing methodologies necessity improvement by application the mechanism updating phase into the lifecycle model. The model of the machines lifecycle, as well as its mathematical and technical-hardware support, was developed on the basis of the carried out research.
\end{abstract}

Nowadays ensuring the maximum possible machines operation is the primary task in the creation of any mechanical system, regardless of its complexity and role. The solution of this problem is to identify factors that have a significant influence on the studied mechanism operational properties. Only by managing each of these factors, it is possible to ensure the operability of any technical system.

Taking into consideration the fact that about $80 \%$ of machines functional losses are connected with the wear of their parts [1], it is necessary to create the conditions that allow the uninterrupted operation of each part to ensure the efficiency of the entire mechanism, it is especially important to pay attention to the most loaded tribocouples.

According to the collected data from various literature sources [2-5], one of the actively developing areas of increasing wear resistance are all kinds of influences on the machines and mechanisms particularly loaded parts surface layer. They distinguish a sufficiently large number of influence ways, such as surfacing, magnetic treatment, mechanical hardening, thermal method, electrophysical, combined, etc. In addition, the work is carried out in the field of lubrication and other promising methods of increasing wear resistance. The application of each of them has a certain influence on tribotechnical properties and, consequently, on the final operability of the entire mechanism. Therefore, it is required to develop the general methodology for selecting the most effective methods and to introduce the most appropriate one depending on the particular mechanism, the influences on it, and the environment where it is situated in.

A probabilistic approach is one of the options for solving the existing problem. It is used to predict the machine working state in a certain time period. This method is realized by constructing a lifecycle model of the most frequently failing conjugation in the form of a transient graph [6] from one phase (situation) to another.
This allows to represent any tribocouple, as a stochastic system that changes its state during the lifecycle. Based on Solomentsev Yu.M., Serdobintsev Yu.P., and others [7-15] surveys, it is possible to assume that any mechanism can be an adaptive system, that is, adapting to a change in load, or to changed external influences. According to this methodology, we proposed the method of the most effective technological solution. Optimization in such a system in accordance with the obtained variables is performed by the optimization criterion. Precisely due to the accuracy of the choice of this criterion, the system will have the highest efficiency. In this connection, we proposed the criterion for choosing the most effective technological solution aimed at ensuring the mechanism operability:

$$
K_{i j}=\min \left|\sum_{k=1}^{m} Z_{i j k} \times H_{i j k} \times P_{i j k}\right|
$$

where, Kij is the resulted criterion estimating efficiency of $\mathrm{j}$-th level $i$-th technology used to ensure the operability;

$\mathrm{k}$ is the number of the phase (situation) arising with the probability Pijk, thus causing the costs Zijk and the wear Hijk; The value Zijk is the ratio of the cost of the machine or mechanism implementing to the resulting technical effect.

The wear is determined by two ways, depending on the geometric dimensions of the part, as well as its purpose and operating conditions. The first way is the so-called method of artificial bases.

According to this method, the holes with a known beforehand geometrical characteristics are cut out on the part's friction surface with a special tool, and their variation is monitored depending on wear. The thickness of the worn layer can be calculated according to the formula [12]:

$$
H_{i j k}=h_{1}-h_{2}=1 / 8\left(l_{1}^{2}-l_{2}^{2}\right) 1 / r
$$

\footnotetext{
* Corresponding author: lyashenko2626@mail.ru
} 
This method is used for parts and conjugations where it is possible to apply bases, without impairing the operational properties of the observed mechanism, for example on different shafts, pistons, etc. In other cases, the use of the labeled atoms method is possible. In this case, a radioactive substance is introduced into the part surface layer, and the wear is determined by measuring sensors, identifying the amount of radiation, detecting radioactive particles in any environment and measuring their quantity, calculating the wear of a particular part.

The mechanism optimization as an adaptive system according to this criterion, in comparison with existing ones, describes more precisely its lifecycle, takes into account the parts actual wear influence, as well as the costs and probability of failure in a given situation, on the technologies effectiveness used to maintain the working capacity.

To determine the tribocouples containment probability in a particular phase (situation) for a given resource, a matrix of transitions from one level to another was compiled. Using the lifecycle model in the form of a transition graph (Fig.1.) and the received matrix, we get:
- the containment probability of a part or a conjunction in the operation phase in the absence of failures:

$$
P_{i j 3, n}=\frac{\sum_{0}^{n} p_{3, n}^{\prime}(3, n-1)+p_{3, n}^{\prime}(5)}{1+\sum_{k=0}^{T-1} p_{k}^{\prime}(k-1)}
$$

where, $\mathrm{T}=7$ is the total number of phases (situations);

-the design phase:

$$
P_{i j 1}=\frac{p_{1}^{\prime}(2)+p_{1}^{\prime}(6)+p_{1}^{\prime}(7)}{1+\sum_{k=0}^{T-1} p_{k}^{\prime}(k-1)}
$$

- the phase of production and promotion to the consumer:

$$
P_{i j 2}=\frac{p_{2}^{\prime}(1)}{1+\sum_{k=0}^{T-1} p_{k}^{\prime}(k-1)}
$$

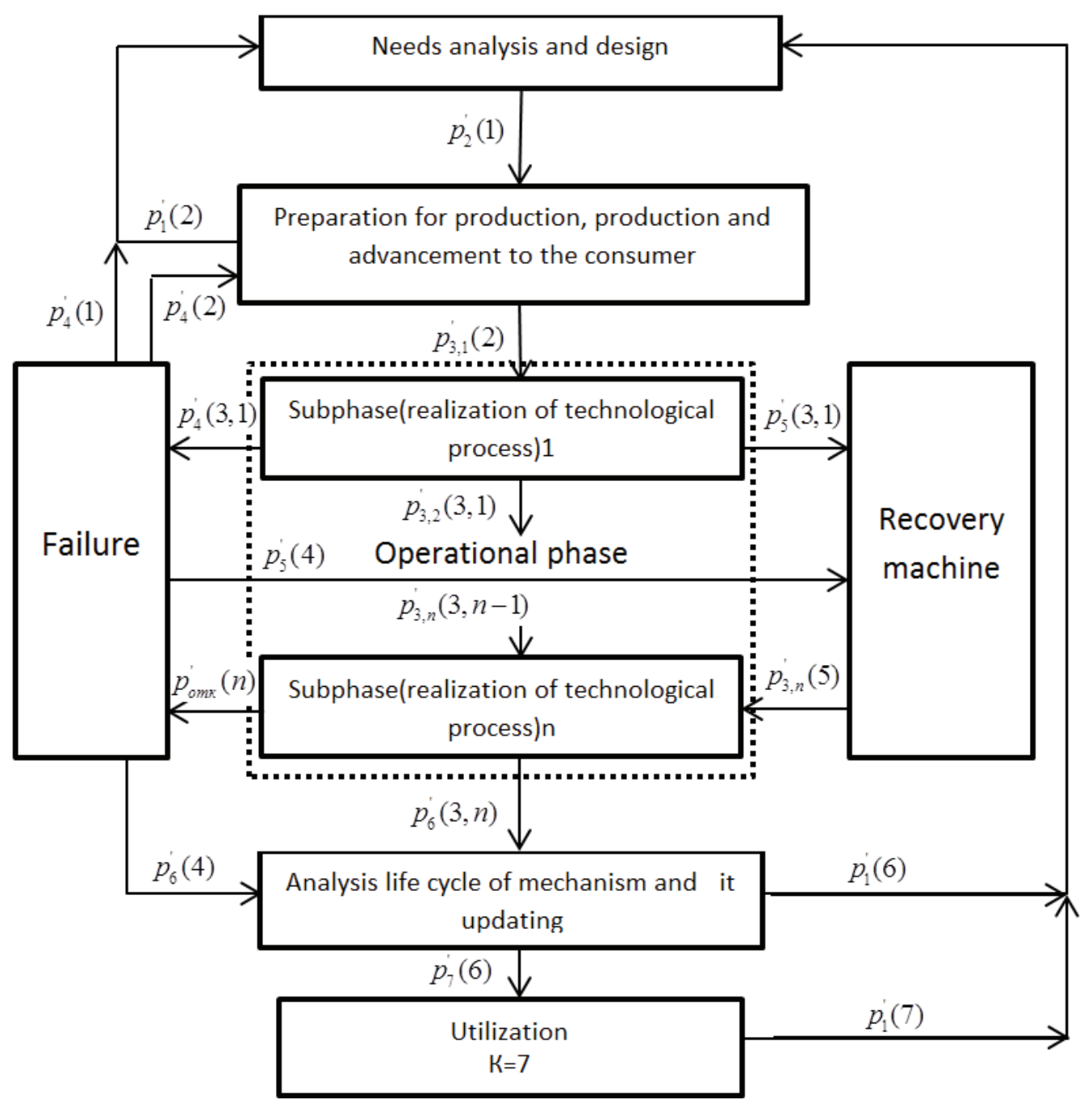

Fig.1. The model of the machine lifecycle due to the improvement phase in the form of a transient graph 
-the failure phase:

$$
P_{i j 4}=\frac{p_{4}^{\prime}(3, n)+p_{4}^{\prime}(1)+p_{4}^{\prime}(2)}{1+\sum_{k=0}^{T-1} p_{k}^{\prime}(k-1)}
$$

-the recovery phase:

$$
P_{i j 5}=\frac{p_{5}^{\prime}(3, n)+p_{5}^{\prime}(4)}{1+\sum_{k=0}^{T-1} p_{k}^{\prime}(k-1)}
$$

-the improvement phase:

$$
P_{i j 6}=\frac{p_{6}^{\prime}(3, n)+p_{6}^{\prime}(4)}{1+\sum_{k=0}^{T-1} p_{k}^{\prime}(k-1)}
$$

-the phase of utilization:

$$
P_{i j 7}=\frac{p_{7}^{\prime}(6)}{1+\sum_{k=0}^{T-1} p_{k}^{\prime}(k-1)}
$$

When varying all the levels of controlled factors, various constraints, depending on the lifecycle phase, were taken into consideration, for example, by operating modes in the operation phase, by mechanical, physical, and technical processing, in the phases of improvement, recovery and production, etc.

As today many mechanical systems are created, each of them has various disadvantages. The example is the responsible tribocouples insufficient wear-resistant surface layer, in the case of its failure further full-scale operation of the entire mechanism is impossible. During this existing models machine failure, it was either restored by any of the known methods, or recycled as a mechanism with an inappropriate repair, and then the lifecycle repeated. The studied models [7-15] did not take into account such factor as the improvement of already created systems, whether parts' laser processing to increase the loaded parts surface layer wear resistance, or magnetic and other kinds of processing for the same purpose - all these methods are widely used in the machines and mechanisms production. And at the moment, when the bulk of scientific works is carried out to develop the already created mechanisms, we cannot neglect such stage in the machine lifecycle as the improvement phase. On its basis, we proposed the model where this factor is the next stage in the lifecycle after the mechanism failure. This allows to consider the failure not as a transition from the operational state to recycling, but rather to provide a failure as the way to improve any mechanical system, and subsequently ensure its higher performance, higher production indices, and in general to increase any machine operational activity, by all possible influences on failing out conjunctions.
In the proposed methodology, the concept of multiloop control of the machines and mechanisms operational properties is considered as the basis, it describes each conjunction of the mechanism throughout the lifecycle. At the same time, the developed methodology allows to realize the functions of the adaptive optimal operability assurance search-free system. In other words, with the help of the described probabilistic methods, already in the initial phases of the mechanism creation, the proposed methodology makes it possible to choose the optimal solution to ensure operability by changing the tribotechnical properties in its parts surface layer. However, under conditions of an infinite number of influences, changing loads, temperature regimes, etc., throughout the operational activity, it is simply impossible to take into account all the influencing factors and their combinations in designing. Therefore, when a machine or mechanism passes through all the lifecycle phases, to solve the set out problem of the subsequent technology application to increase the wear resistance of the failed tribocouples surfaces, we need the information on all the influences that this mechanism experiences in a particular environment. Collected with the help of pre-installed, automated sensors, information will allow to correct the previously adopted technological influences and to implement adaptive control. We propose to combine the applicable sensors in a single diagnostic system with automatic data collection. This approach to monitor the mechanism parts actual state will reduce the number of failures, and subsequently, save the time that is usually spent on the machine recovery after the failure.

In the developed methodology, the structure of information support for choosing one of the alternative technological influences and their parameters for failing tribocouples corresponds to the algorithm of stage-bystage access to resources of various levels in the process of ensuring the overall operability of the entire mechanism. (Fig.2.).

This block diagram [14] is suitable for optimized accepting of technological solutions in various mechanisms adaptive control.

To ensure selection of the most effective application of technological influences on the analyzed mechanism, all data, received from the sensors and other tracking devices, was saved in database. It has a hierarchical structure, and is based on characteristic equation [15] describing all the signs and characteristics of controlled mechanical system. It includes the values of contact interaction parameters, the calculated values, the values obtained from automatic sensors, in this case all sorts of situations under their serial numbers and valid values for required performance properties to ensure efficiency in accordance with degree of each component responsibility and its functionality entered in the database.

The implementation of the system for the selection effective technological solutions, and, consequently, to ensure operation of mechanical systems is carried out by our proposed scheme (Fig.3.). 


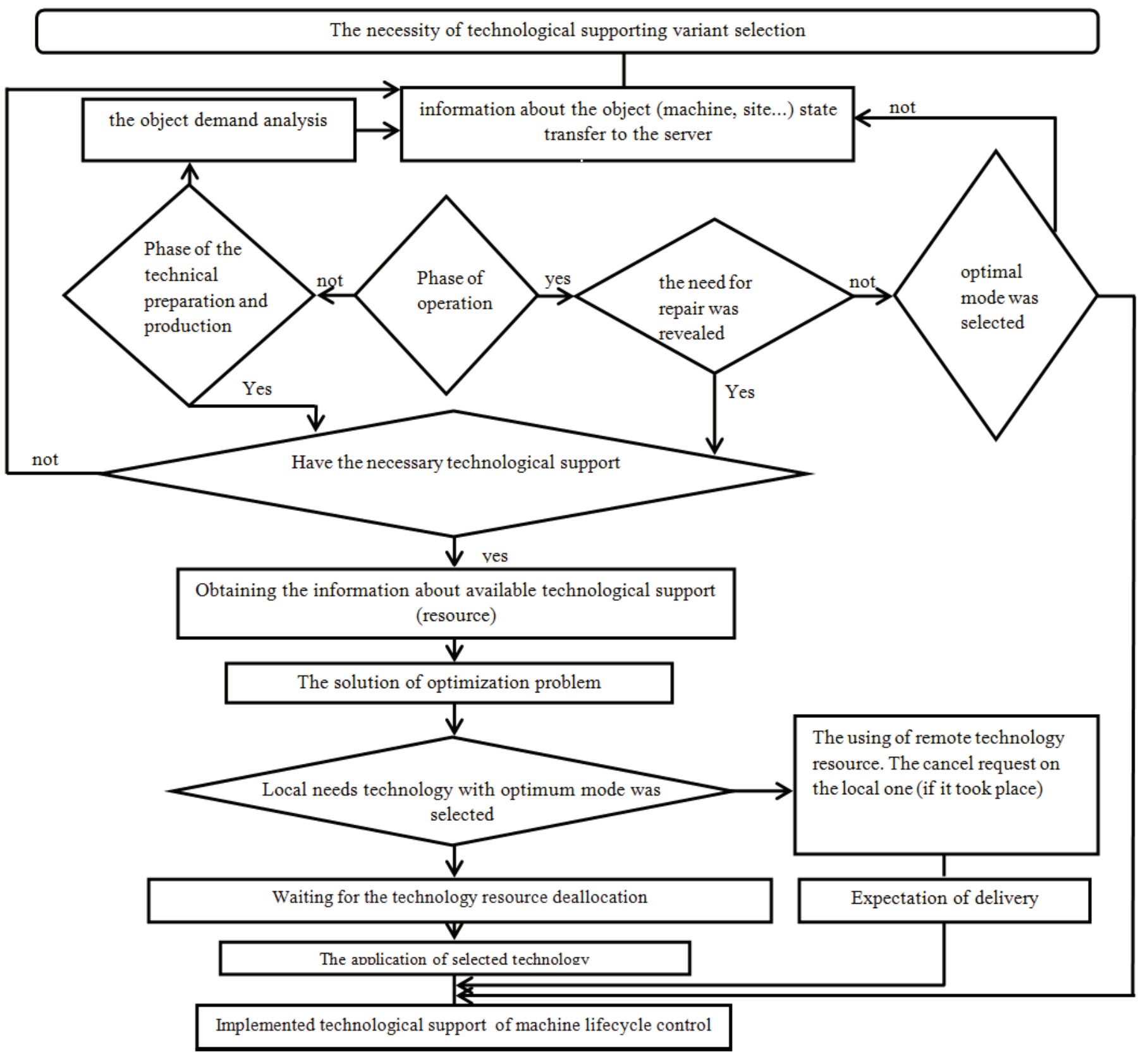

Fig.2. Algorithm of machine lifecycle control technological support

This scheme was developed on the basis of the operation [15] and improved by making new components for the machine performance maintaining.

We considered machine as the system consisting of many components and conjunctions that with certain probability can fail from time to time in the machine lifecycle. In this case, after failure, the perfection of mechanism is carried out by the means of one type strengthening surface processing use taking account factors, which leaded to the failure.

The proposed scheme consists of technical means aimed at diagnosis and monitoring of wear values in comparison with values from the database, and selection and implementation of specific technology to improve damaged tribocoupling, in accordance with received data. Mathematical integration of separate subsystems into a single hierarchical control system structure is the apparatus of queueing theory.

Thus, we developed the methodology of options and parameters for technological support evaluation, which allows to use the most effective technological solution according to the criterion, which includes technical and economic indicators. The improved lifecycle mechanism model including the process of improving and modification broken machine parts and components was put into the basis of proposed methodology.

In accordance with the proposed lifecycle machine model as adaptive systems, we chose the optimization criterion including factors that most broadly cover the lifecycle of any mechanism, which means better reflecting technical condition of system under study. This criterion allows to make right decisions on applications technologies improving wear resistance of 
mechanisms. Our proposed optimization criterion includes the ratio of each part deterioration, the cost of their creating, recovery and improvement, as well as probability of failure depending on lifecycle phase, where the considered mechanism is situated.

Additionally, we chose the algorithm of the machine lifecycle management technological support and designed the structural scheme of technical-hardware support of mechanical systems performance, where we tried to cover the process of machines and mechanisms work capacity supporting.

This approach, unlike existing, allows to apply the most effective methods of various tribocouples friction surfaces improving, to increase performance of mechanical system, based on the data obtained during the entire lifecycle of the studied mechanism. At the same time, taking into account the mechanism technical state constant monitoring, it allows to correct the decision taken earlier, depending on the changing operating conditions and the actual state of the machine components and parts.

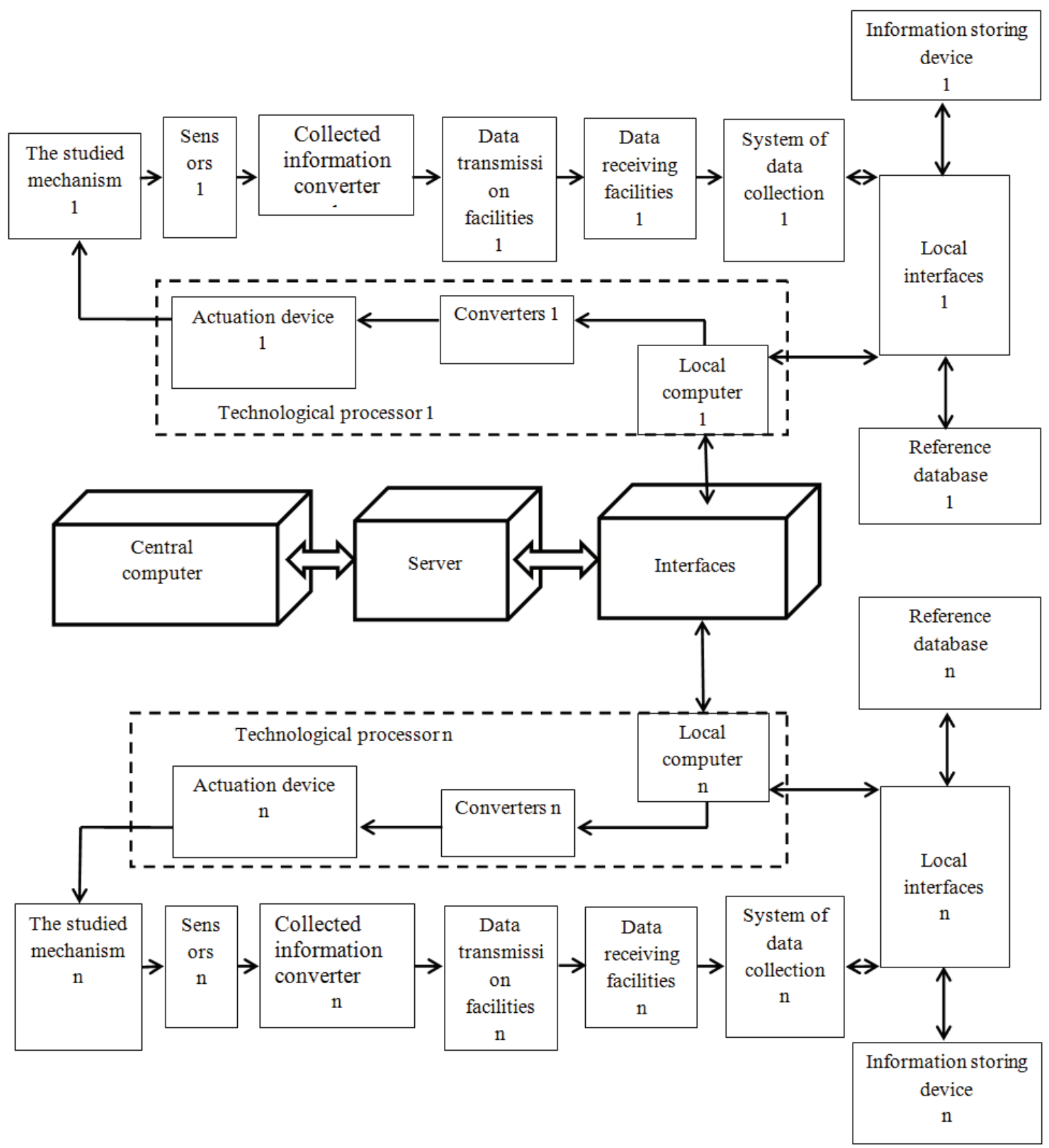

Fig.3. The generalized block diagram of technical-hardware support of mechanical systems performance 


\section{References}

1. Zh.A. Popova, BSU Herald, 4, 43-46 (2012).

2. I.V. Kragelskii, Friction and Wear, (Butterworths, London, 1965).

3. B. I. Kostetskii, I. G. Nosovskii, L. I. Bershadskiy, A. K. Karaulov, Reliability and durability of the machines (Techque, Kiev, 1975).

4. O.V. Burlachenko, M.V. Ivanov, Proc. Eng. 150, 464-467 Elsev. Publ. (2016).

5. B. I. Kostetskii, Friction, lubrication and wear in machines, (Technique, Kiev, 1970).

6. V.E. Gmurman, Probability theory and mathematical statistics, 8 (Higher School, Moscow, 2002).

7. B.N. Orlov, A.I. Novichenko, N.B. Orlov, Environ. Engin., 4, 88-90 (2015).

8. Yu. M. Solomencev, R.R. Zagidullin, E.B. Frolov, FRC CSC RAS, 4, 77-87 (2010).

9. A.N. Maksimenko, Bull. of BRU, 2, 31-40 (2012).

10. O.V. Burlachenko, Technological support machine performance (VSUAE, Volgograd, 2002).

11. A.V. Zazykin, S. V. Repin, N. K. Hovalyg, Bull. of Civ. Eng., 1,85-94 (2009)

12. A. S. Pronikov, Reliability of the machines, (Mechanical engineering, Moscow, 1978).

13. V. I. Atopov, Y. P.Serdobintsev, O. K. Slavin, Modeling of contact stresses, (Mechanical engineering, Moscow, 1988).

14. Y.P. Serdobintsev, O.V. Burlachenko, A.G. Skhirtladze, Improving the quality of functioning of technological equipment, (TNT, Stary Oskol, 2010).

15. O.V. Burlachenko, Affordable and comfortable housing to Russian citizens, VSUASE, 65-67 (2009). 